\title{
Influence d'un fluide annulaire sur les vitesses critiques d'un arbre en rotation
}

\author{
Effect of the surrounding fluid on critical speeds of a rotating shaft \\ Joël Guidez \\ Christian Girard \\ DRNR/STRS - CEN/Cadarache \\ DRE/STRE - CEN/Cadarache
}

\section{Paul Jarriand}

Société Neyrpic - Grenoble

\section{Notations utilisées}

$\phi$ : fonction d'onde

$C$ : vitesse du son dans le fluide

$L$ : longueur de l'arbre (ou entraxe entre les 2 paliers)

$r, \theta, z$ :coordonnées cylindriques

$\omega: \quad$ vitesse de rotation

$R_{i}$ : rayon extérieur de l'arbre

$R_{e}$ : rayon intérieur de la virole

$P: \quad$ Pression

$M$ : masse

$\rho_{o}:$ masse volumique du fluide

$M^{\prime}$ : masse ajoutée

$K: \quad$ raideur

$M_{S}:$ masse de Stockes

$k: \quad$ paramètre de confinement

$f_{\text {air }}$ : fréquence propre en air

$f_{\text {fluide }}:$ fréquence propre en fluide

\section{Introduction}

Les pompes primaires des réacteurs rapides (fig. 1) ont un arbre vertical de longueur importante guidé par 2 paliers

- un palier supérieur à roulements

- un palier hydrostatique inférieur en sodium.

Cet arbre est entouré d'une virole servant à la fois :

- de guide d'obturateur

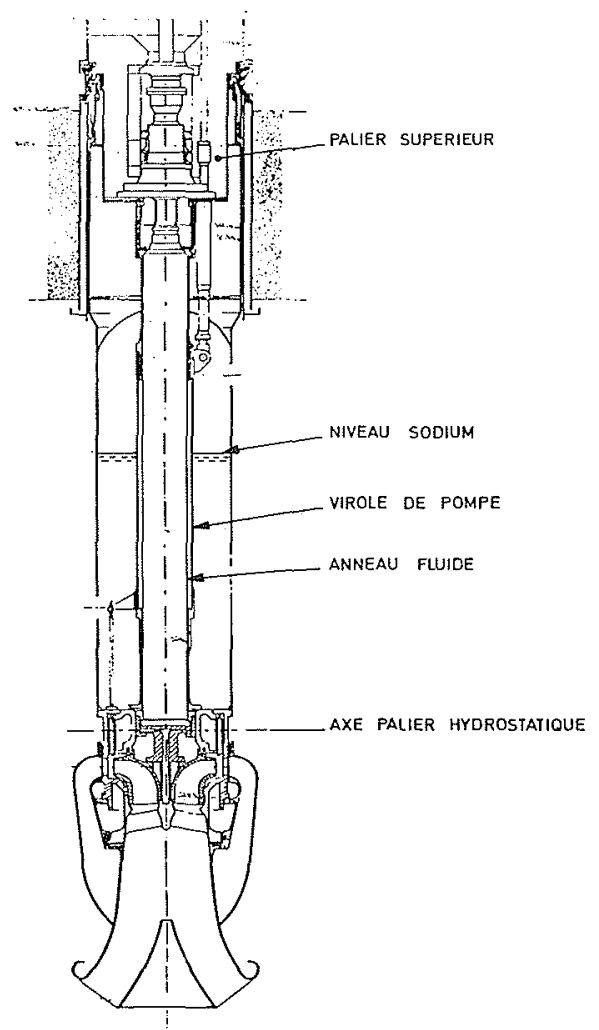

Figure 1 - Exemple de type de pompe primaire pour un réacteur rapide.

- de limiteur du vortex induit dans le fluide par la rotation de l'arbre.

Le fluide compris entre cette virole et l'arbre a deux effets sur les vitesses critiques de l'arbre :

1) un effet de masse ajoutée qui tend à les diminuer

LA HOUILLE BLANCHE/N ${ }^{\circ} 1 / 2-1980$ 
2) un effet d'amortissement lors du passage de ces vitesses.

Le but de cet exposé est d'étudier et de quantifier ces phénomènes physiques de manière à pouvoir :

- dimensionner un arbre sous-critique en tenant compte de l'effet de masse ajoutée

- envisager la réalisation d'arbre hypercritique utilisant ces phénomènes.

Cette analyse est effectuée, d'abord de manière théorique, puis grâce aux résultats d'essais effectués sur un arbre de $1 \mathrm{~m}$ chez Neyrpic en 1978.

\section{Etude théorique du problème}

Si l'on suppose que les oscillations d'un arbre immergé sont petites, les mouvements du fluide peuvent être décrits par l'équation d'onde qui, en coordonnées cylindriques, s'écrit :

$$
\frac{1}{r} \frac{\partial}{\partial r}\left(r \frac{\partial \phi}{\partial r}\right)+\frac{1}{r^{2}} \frac{\partial^{2} \phi}{\partial \theta^{2}}+\frac{\partial^{2} \phi}{\partial z^{2}}-\frac{1}{C^{2}} \frac{\partial^{2} \phi}{\partial t^{2}}=0
$$

[Ref. 2]

$C$ : vitesse du son dans le fluide

$\phi$ : fonction d'onde

Dans le cas où l'on néglige l'écoulement axial, la solution est du type :

$$
\phi(r, \theta, t)=\varphi_{(r)} \cos . \theta \cdot e^{i w t}\left(\frac{\partial \phi}{\partial_{z}}=0\right)
$$

En reportant dans (1) on obtient :

$$
\frac{\partial^{2} \varphi}{\partial r^{2}}+\frac{1}{r} \frac{\partial \varphi}{\partial r}+\left[\frac{w^{2}}{C^{2}}-\frac{1}{r^{2}}\right] \varphi=0
$$

Equation dont la solution est donnée par les fonctions de Bessel :

$$
\varphi_{(r)}=A J_{1}\left(\frac{w r}{C}\right)+B Y_{1}\left(\frac{w r}{C}\right)
$$

Les constantes $A$ et $B$ seront alors déterminées par les conditions aux limites

a) sur la paroi du cylindre intérieur $\left(R_{i}\right)$

$$
\frac{\partial \varphi}{\partial r}\left(r=R_{i}\right)=i w x_{o}
$$

$\left(x_{0}=\right.$ amplitude de la vibration)

b) sur la paroi du cylindre extérieur $\left(R_{e}\right)$

$$
\frac{\partial \varphi}{\partial r}\left(r=R_{e}\right)=0 \text { (cas sans couplage) }
$$

Connaissant la fonction d'onde $\phi$ nous savons que :

$$
P(r, \theta, t)=-\rho_{o} \frac{\partial \phi}{\partial t}
$$

( $p$ : pression dans le fluide)
D'où par intégration pour $r=R_{i}$ nous obtenons la force à laquelle est soumis le cylindre intérieur :

$$
F=-\int_{S} \cdot \rho_{o} \frac{\partial \phi}{\partial t} d S
$$

Dans notre cas (basses fréquences) nous avons :

$$
\frac{w R_{i}}{C} \text { et } \frac{w R_{e}}{C}<1
$$

On peut alors écrire :

$$
F=\pi L \rho_{o} R_{i}^{2} \frac{R_{e}^{2}+R_{i}^{2}}{R_{e}^{2}-R_{i}^{2}} w^{2} x_{o}
$$

L'équation du mouvement du cylindre intérieur est de la forme :

$$
-M x^{*}+K x=F
$$

$\mathbb{I}$ vient alors avec $x=x_{o} e^{i w t}$

$$
-\left[M+\pi L \rho_{o} R_{i}^{2} \frac{R_{e}^{2}+R_{i}^{2}}{R_{e}^{2}-R_{i}^{2}}\right] w^{2} x_{o}+K x_{o}=0
$$

On voit donc que dans le cas d'un fluide confiné entre 2 cylindres (sans appuis et sans couplage), la présence du fluide fait intervenir une masse supplémentaire appelée masse ajoutée de la forme :

$$
M^{\prime}=\pi L \rho_{o} R_{i}^{2} \cdot \frac{R_{e}^{2}+R_{i}^{2}}{R_{e}^{2}-R_{i}^{2}}
$$

\section{Cas de l'arbre sur 2 appuis}

On n'a plus alors $d \phi / d_{z}=0$.

On écrit alors en supposant une variation sinusoidale de la fonction d'onde

$$
\phi(r, \theta, z, t)=\varphi_{(r)}-\sin \left(\frac{n \pi}{L} z\right) \cdot \cos \theta e^{i w t}
$$

Il vient alors tout calcul fait une masse ajoutée de la forme :

$$
M^{\prime}=\frac{8}{3} L \rho_{o} R_{i}^{2} \frac{R_{e}^{2}+R_{i}^{2}}{R_{e}^{2}-R_{i}^{2}}
$$

$$
\text { (cas où } \frac{\pi R_{i}}{L}, \frac{\pi R_{e}}{L}<<1 \text { ) }
$$

En conclusion il apparait que le mode propre du système est modifié et nous avons pour le mode $n$.

$$
f_{n}^{\prime}=\sqrt{\frac{K_{n}}{M_{n}+M_{n}^{\prime}}}
$$

$K_{n}$ : raideur généralisée

$M_{n}$ : masse généralisée pour le mode $n$ en air

$M_{n}^{\prime}$ : masse ajoutée projetée sur le mode $n$ en présence de fluide.

Posons :

$$
M_{S}=\pi L \cdot R_{i}^{2} \rho_{o}
$$


$M_{S}$ est alors la masse de Stockes, c'est-à-dire la masse ajoutée à prendre en compte pour un cylindre infiniment long dans un fluide sans confinement :

$$
\text { Posons } k=\frac{R_{e}^{2}+R_{i}^{2}}{R_{e}^{2}-R_{i}^{2}}
$$

$k$ est alors le paramètre faisant intervenir le confinement du fluide.

La masse ajoutée aura alors comme expression :

$$
M_{n}^{\prime}=\alpha \cdot k \cdot M_{S}
$$

$\alpha$ étant alors un coefficient tenant compte des phénomènes perturbateurs (appuis, rotation, etc.).

On aura donc :

$$
\frac{w_{n}}{w_{n}^{\prime}}=\sqrt{1+\frac{\alpha k M_{S}}{M_{n}}}
$$

C'est à l'aide de cette formule simple que nous allons interpréter les essais effectués et en particulier analyser l'influence des phénomènes perturbateurs sur le terme $\alpha$.

\section{Présentation des essais}

Les essais étaient effectués sur un arbre de longueur $1 \mathrm{~m}$, de diamètre $70 \mathrm{~mm}$ monté sur roulements (fig. 2).

Les essais se sont déroulés dans un esprit d'étude paramétrique, soit :

- avec différentes montées en vitesse

- avec 3 viroles de confinement ( $\phi 80, \phi 125, \phi 210)$

- avec 2 viroles de $\phi 80$ de raideur différente (phénomène de couplage)

- avec des fluides de densité et de viscosité différente

- en faisant varier le niveau libre du fluide dans l'entrefer.

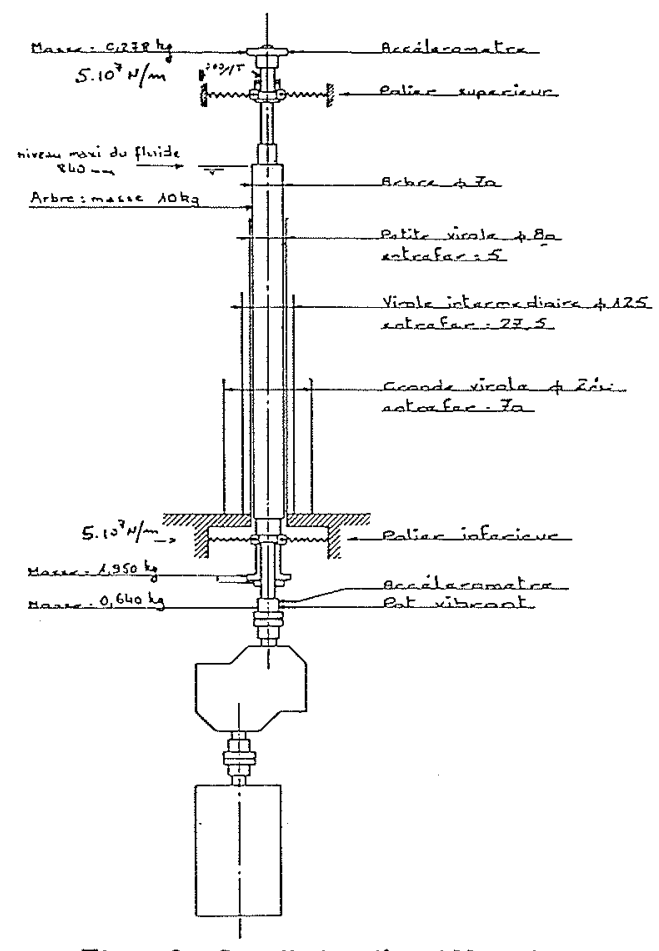

Figure 2 - Installation d'essai Neyrpic.

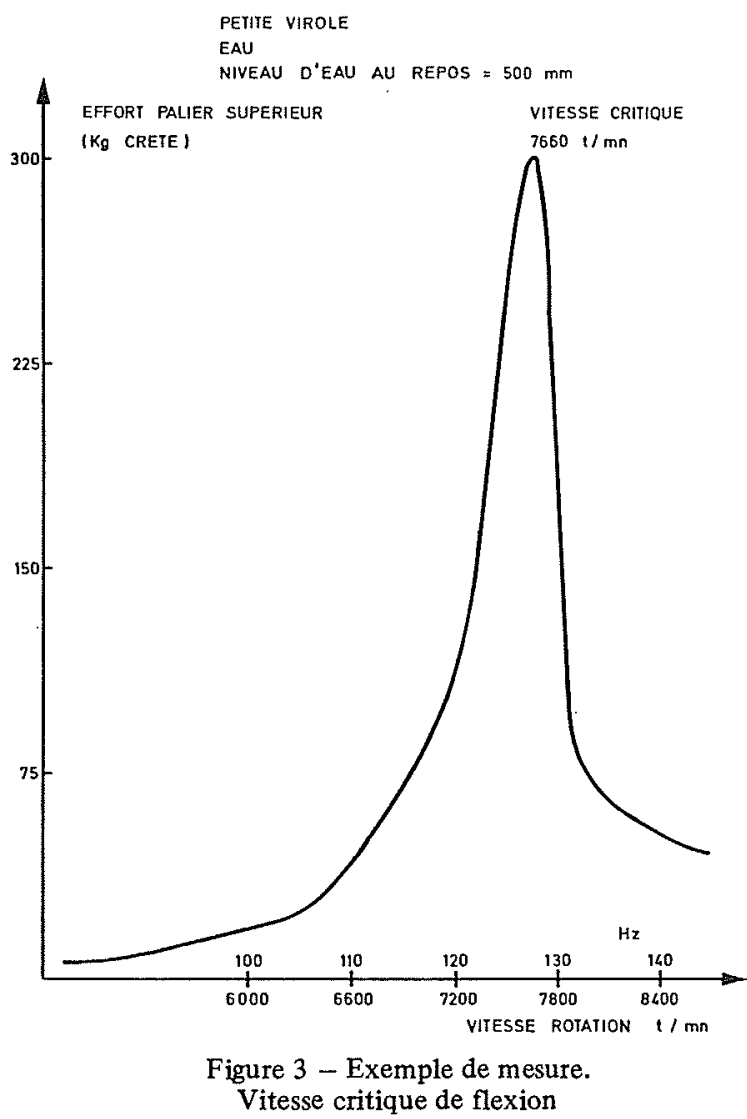

Pour tous ces cas on a effectué des passages de la première vitesse critique en mesurant l'évolution des efforts au niveau des roulements (fig. 3) en fonction de la vitesse.

Une campagne de mesure équivalente a été effectuée arbre au repos avec un pot vibrant.

Toutes les mesures ont été gardées sur bande magnétique pour dépouillement ultérieur (fig. 6).

Analyse des résultats et comparaison avec la théorie

\section{Rappel}

Tous les essais seront interprétés par la formule,

$$
\frac{f_{\mathrm{air}}}{f_{\text {fluide }}}=\sqrt{1+\frac{\alpha k M_{S}}{M}}
$$

On s'efforcera de voir l'influence des termes pertubateurs,

- appuis,

- rotation du fluide,

- dénoyage par le vortex etc...

sur la valeur de $\alpha$.

\section{Influence de la masse volumique}

La masse ajoutée devrait être proportionnelle à la masse volumique du fluide $\rho_{0}$. 
On aurait donc,

$$
\frac{f_{\text {air }}}{f_{\text {fluide }}}=\sqrt{1+k^{\prime} \rho_{0}}
$$

Les résultats expérimentaux le prouvent.

Exemple : Essais en rotation avec petite virole,
Vitesse critique dans l'air
Vitesse critique en eau
: $8000 \mathrm{tr} / \mathrm{mn}$
: $5170 \mathrm{tr} / \mathrm{mn}$
Vitesse critique en huile $(\rho=1,45): 4600 \mathrm{tr} / \mathrm{mn}$
On trouve,
$\mid \begin{array}{ll}\text { pour l'eau } & K^{\prime}=1,394.10^{-3} \\ \text { pour l'huile } & k^{\prime}=1,39610^{-3}\end{array}$

\section{Application :}

La masse ajoutée en sodium sera 0,85 fois plus faible qu'en eau.

\section{Influence de la montée en vitesse}

L'essai a été effectué avec la petite virole entrefer $5 \mathrm{~mm}$ avec niveau $840 \mathrm{~mm}$ constant.

\begin{tabular}{|c|c|c|c|}
\hline $\begin{array}{c}\text { Montée en } \\
\text { vitesse }\end{array}$ & $\begin{array}{c}\text { Vitesse de } \\
\text { balayage } \\
\text { (tr/mn/sec) }\end{array}$ & $\begin{array}{c}\text { Apparition } \\
\text { de la vitesse } \\
\text { critique }\end{array}$ & $\begin{array}{c}\text { Amplitude } \\
\text { Effort palier } \\
\text { (kg créte) }\end{array}$ \\
\hline 4000 à $5500 \mathrm{tr} / \mathrm{mn}$ & 7,5 & $5170 \mathrm{tr} / \mathrm{mn}$ & 37 \\
0 à $7540 \mathrm{tr} / \mathrm{mn}$ & 37,5 & $5170 \mathrm{tr} / \mathrm{mn}$ & 37 \\
0 à $7540 \mathrm{tr} / \mathrm{mn}$ & 75,4 & $5170 \mathrm{tr} / \mathrm{mn}$ & 37 \\
0 à $7540 \mathrm{tr} / \mathrm{mn}$ & 150,8 & $5270 \mathrm{tr} / \mathrm{mn}$ & 37 \\
0 à $7540 \mathrm{tr} / \mathrm{mn}$ & 377 & $5312 \mathrm{tr} / \mathrm{mn}$ & 37 \\
\hline
\end{tabular}

On retrouve de manière qualitative un retard à l'apparition de la $1^{\text {ere }}$ vitesse critique pour les plus fortes montées en vitesse.

Nos vitesses de balayage n'étaient cependant pas suffisantes pour enregistrer un retard plus important de cette apparition, lié à une diminution de l'amplitude des efforts.

\section{Aspect qualitatif du phénomène}

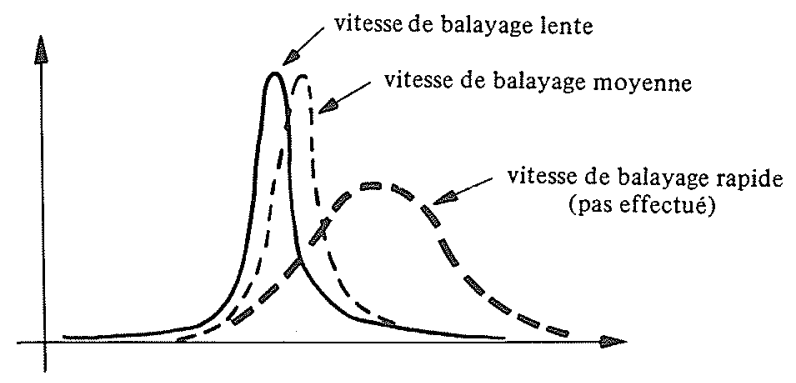

Ce phénomène est utilisé industriellement pour certains rotors hypercritiques. Nous n'avons cependant pas poursuivi dans cette voie, les vitesses de montée correspondantes n'étant pas compatibles avec celles exigées au niveau d'un réacteur rapide.

\section{Influence du niveau libre}

On a reporté sur la figure 4 les rapports $f_{\text {fluide }} / f_{\text {air }}$ dans le cas des 3 viroles pour différents niveaux libres.

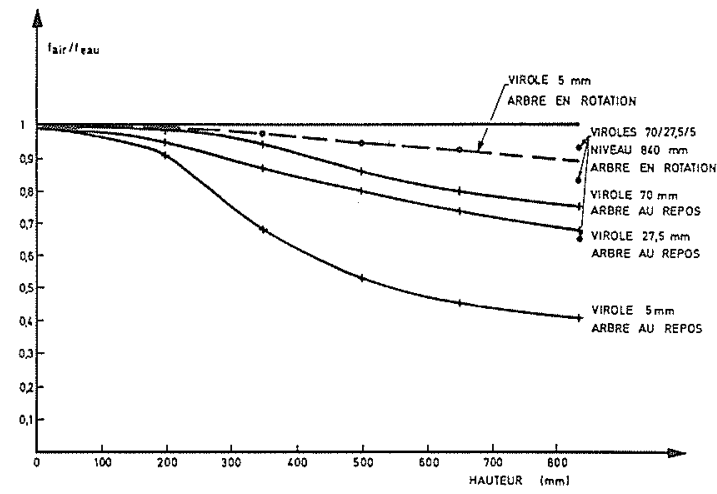

Figure 4 - Graphe récapitulatif des principaux points de mesure.

Les mesures ont été effectuées arbre au repos par pot vibrant.

N.B. : On sait en effet qu'à certaines approximations près (effet gyroscopique négligé) on peut assimiler la valeur de la $1^{\text {ere }}$ vitesse critique à une fréquence propre de flexion.

\section{Vérification de la formule (2)}

Le calcul de $\alpha_{M_{S}} / M$ pour les différents cas donne :

\begin{tabular}{|c|c|c|c|}
\hline Niveau & Petite virole & Virole movenne & Grande virole \\
\hline 840 & 0,634 & 0,622 & 0,605 \\
650 & 0,485 & 0,444 & 0,44 \\
500 & 0,3 & 0,274 & 0,276 \\
350 & 0,129 & 0,168 & 0,095 \\
\hline
\end{tabular}

On retrouve bien à chaque niveau $\alpha_{M_{s}} / M$ constant.

On a donc bien $\alpha$ indépendant du confinement. $L a$ constante $k$ représente donc bien l'influence $d u$ confinement sur la masse ajoutée.

En fait $\alpha$ représente la modification du mode lorsque la barre vibre en mode 1 en présence de fluide.

Si $X_{1}$ représente le mode 1 de la barre dans l'air, et si $Y_{1}$ représente le mode 1 de la barre dans le fluide, le rapport $\alpha$ représente,

$$
\frac{\int_{0}^{L} y_{1}^{2} d x}{\int_{0}^{L} x_{1}^{2} d x}
$$

Ce rapport n'est pas modifié par le confinement mais par la hauteur (1) de fluide car,

- d'une part l'intégration se fait de 0 à 1 (et non à $L$ )

- d'autre part le mode $Y_{1}$ est fonction de la hauteur d'eau.

Enfin on peut noter que toutes ces valeurs de fréquences propres mesurées lors des essais ont été retrouvées à l'aide du code de calcul Tedel Fluide avec une marge d'erreur faible.

\section{Influence de la vitesse de rotation}

Nous supposons dans ce chapitre que le niveau de fluide reste au niveau maxi. Le fluide est alors entrainé en rotation par l'arbre. 
Pour de faibles épaisseurs de fluide, on peut montrer sous certaines hypothèses [Réf. 1] que la masse ajoutée due au fluide est alors divisée par un facteur 4 .

Pour les épaisseurs importantes le régime est totalement turbulent et l'approche théorique du phénomène difficile.

On a reporté sur la figure 4 les 3 points de mesure correspondant aux 3 viroles pour un niveau 840 , arbre en rotation.

Le calcul donne alors dans les 3 cas,

$\mid \begin{array}{ll}\alpha^{\prime}=\alpha / 4 & \text { (petite virole) } \\ \alpha^{\prime}=\alpha / 3 & \text { (virole moyenne) } \\ \alpha^{\prime}=\alpha / 4,7 & \text { (grande virole) }\end{array}$

On propose donc de garder en lère approximation le rapport de réduction de la masse ajoutée de $1 / 4$ tout en sachant que cette valeur n'est plus rigoureuse pour les épaisseurs de fluide importantes (régime de Taylor ou Régime turbulent).

\section{Influence du vortex}

Pour les moyennes et grandes viroles, les essais avec niveau libre conduisaient en rotation à un dénoyage de l'arbre avant d'atteindre la première vitesse critique.

On a donc reporté sur la figure 4 les points correspondant uniquement à l'essai en rotation avec petite virole.

Le niveau libre correspondant indiqué est le niveau à l'arrêt.

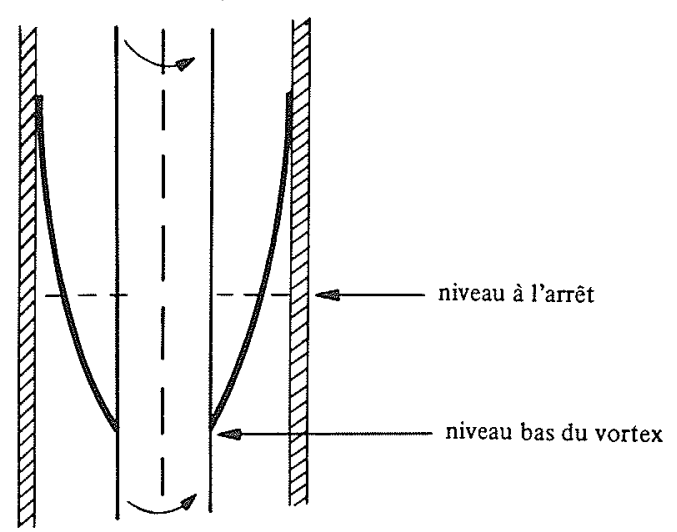

On voit que la courbe obtenue ne passe pas par le point correspondant au niveau 840 sans vortex.

Des calculs approximatifs montrent alors que le niveau bas du vortex doit se trouver à 8000 tr aux environs de $300 \mathrm{~mm}$, pour un niveau à l'arrêt de $840 \mathrm{~mm}$.

Cette valeur correspondant à peu près à la valeur obtenue pour $300 \mathrm{~mm}$, arbre au repos, avec coefficient de réduction de $1 / 4$ à la rotation, ou en conclura en $1^{\text {ere }}$ approximation que lorsque l'arbre est en rotation seule la partie du fluide sous le niveau bas du vortex intervient dans l'expression de la masse ajoutée.

\section{Couplage de la virole extérieure}

Pour des viroles de petit diamètre il y a risque de couplage de la virole extérieure avec l'arbre par l'intermédiaire du fluide.

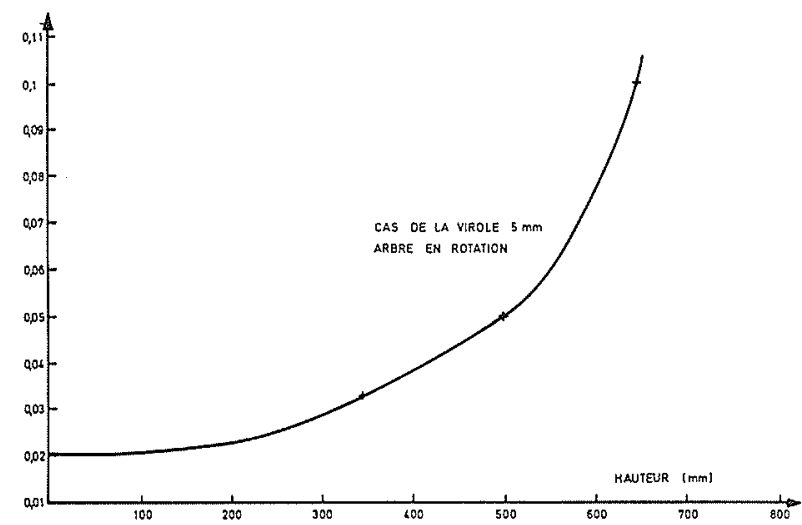

Figure 5 - Evolution du coefficient d'amortissement avec le niveau libre.

Un essai a été effectué avec 2 petites viroles de raideur différentes, qui a permis de retrouver les valeurs obtenues théoriquement. L'abaissement de fréquence dû au couplage restait par ailleurs dans notre cas relativement faible.

\section{Amortissement}

On a reporté sur la figure 5 les valeurs d'amortissement obtenues lors de l'essai en rotation avec petite virole.

Il apparait que ces valeurs augmentent notablement avec le niveau.

De plus alors que ces valeurs sont très faibles ou négligeables pour un arbre au repos, elles semblent devenir importantes pour un fluide en rotation, lorsque celui-ci est d'épaisseur relativement faible.

L'étude du phénomène n'a pas été poussée plus loin dans ces essais, et a été reportée aux essais $2^{\mathrm{eme}}$ phase (voir conclusion).

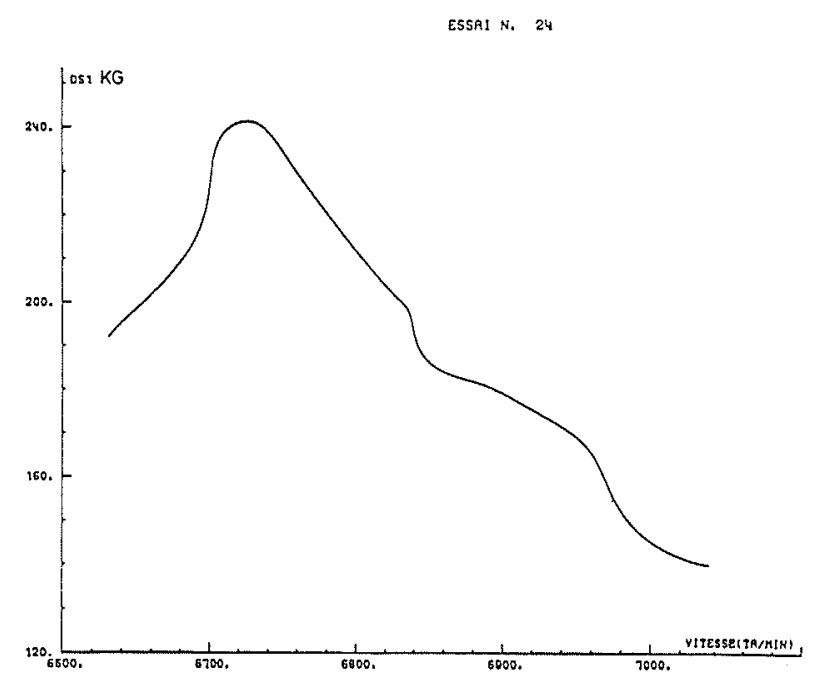

Figure 6 - Exemple de dépouillement d'une réponse du capteur supérieur. 


\section{Application}

\section{Calculd'un arbre sous critique}

Des observations ci-dessus, se dégage une méthode de calcul permettent d'apprécier l'influence du fluide confiné sur l'abaissement de la $1^{\text {ere }}$ vitesse critique.

1) Calcul de la $1^{\text {ere }}$ vitesse critique sans tenir compte du phénomène.

2) Estimation du niveau bas du vortex pour cette vitesse dans la géométrie donnée.

3) Calcul pour ce niveau de fluide, de la masse ajoutée correspondante (ou de la nouvelle vitesse critique par Tedel Fluide) avec fluide au repos.

4) Application du terme correcteur de $1 / 4$ sur la masse ajoutée obtenue pour tenir compte de l'effet de rotation.

5) Vérification des problèmes de couplage avec les structures fixes.

En tout état de cause pour un arbre vertical à niveau libre, l'effet

- du terme réducteur de $1 / 4$,

- du dénoyage dû au vortex

conduit souvent à ramener l'effet de masse ajoutée du fluide à des valeurs inférieures à $10 \%$

\section{Arbre hypercritique}

L'augmentation importante du coefficient d'amortissement lorsque l'arbre est en rotation nous permettait lors des essais, non seulement de passer la vitesse critique, mais même de rester de manière stationnaire sur cette première vitesse critique pour des niveaux libres de fluide suffisamment importants dans la petite virole.
Cet effet semble donc encourageant pour poursuivre l'étude ultérieure d'un arbre hypercritique utilisant ce phénomène.

\section{Conclusion}

L'essai effectué a permis de retrouver certaines données théoriques.

L'interprétation, qui en est faite, permet -sous certaines approximations - de posséder une méthode de calcul de l'abaissement de vitesse critique induit par le phénomène.

De nombreuses inconnues restent cependant encore à lever.

Citons par exemple:

- justification de l'influence du fluide en rotation,

- estimation plus précise du vortex et de son influence,

- évolution des facteurs d'amortissement.

Dans cet esprit un essai $2^{\text {ème }}$ phase a donc été lancé au CEN/Cadarache.

Cet essai, effectué dans le même esprit avec un arbre de $5 \mathrm{~m}$, reproduisant un arbre de réacteur quant aux appuis, devrait permettre :

- de valider la méthode de calcul

- de conduire la solution arbre vertical hypercritique à un stade pré-industriel.

\section{Références}

[1] FRITZ R.J. - The effect of liquid on the dy namic notions of immersed solids, Journal of engineering for industry, Février 72.

[2] LANDAU-LIFSHITZ. - Fluid mechanics. Pergamon Press. 


\section{Discussion}

Président : M. P. BERGERON

M. le Président. - Quelqu'un souhaite-t-il intervenir sur cette communication?

M. MILAN. - Comment s'explique physiquement la division par 4 de la masse ajoutée lorsque le fluide est en rotation.

M. GUIDEZ. - La comparaison qualitative entre la fréquence propre de flexion d'un arbre entouré de fluide immobile et le passage d'une vitesse critique au fluide en rotation n'est pas simple. Ce travail a été effectué dans l'article de M. FRITZ cité en référence dans la communication.

M. GILMER. - L'application de ces formules comporte un piège lorsqu'on approche du rapport 1 entre les deux diamètres. Lorsque l'entrefer est très faible, la vitesse critique réelle peut être de $30 \%$ supérieure à la vitesse critique calculée. Pour une pompe multicellulaire, il a suffi de rainurer circonférentiellement le tambour d'équilibrage pour annuler cet effet. Ordre de grandeur du diamètre du tambour : $300 \mathrm{~mm}-$ jeu : $0,3 \mathrm{~mm}$.

M. GUIDEZ. - Les formules données ne sont valables que pour des entrefers où il n'y a pas d'effèt hydrodynamique de portance.

On peut également rappeler pour les petits entrefers les problèmes de couplage avec la virole.

M. GIRARD. - Avec des jeux de cet ordre, on crée alors un $3^{\text {ème }}$ palier, d'où remontée logique de la vitesse critique.

M. GUIDEZ. - Le but de la formulation proposée est, pour les fabricants ayant des pompes verticales avec niveau libre, de retrouver l'effet de masse ajoutée du fluide entre arbre et virole avec entrefer assez important.
M. CAILLOT. - Dans ce type de pompe, n'y a-t-il pas une influence de la structure porteuse extérieure sur les vibrations propres de l'arbre?

M. GUIDEZ. - Pour la pompe, linfluence de la structure extérieure est très nette. Il faut tenir compte de cette influence pour étudier correctement le problème.

M. HUFFENUS. - Je voudrais revenir sur cette question de surface libre qui a été traitée de façon raisonnable mais cependant très imparfaite puisque le modèle réduit hydroélastique ne permet pas de respecter le nombre de Froude et introduit ainsi une distorsion considérable de la surface libre représentée sur le modèle. (Ainsi la dénivellée occasionnée par la rotation de l'arbre est pratiquement de l'ordre de grandeur de la hauteur du modèle).

Il est bien sûr raisonnable de penser que seule la partie du fluide en contact direct avec l'arbre participe à l'effet de masse ajoutée et d'amortissement, si bien que l'on peut, peut-être, se passer d'une représentation à l'échelle de la surface libre. Encore faut-il que cette surface libre soit parfaitement stable en sorte que la cote du contact fluide-arbre ne varie pas. Qu'en est-il réellement?

M. GUIDEZ. - En effet, pour des vitesses de rotation importantes il y a formation "d'émulsion" en partie haute peu assimilable à une surface libre. Une étude hydraulique complète des problèmes de stabilité de Vortex entre arbre en rotation et virole reste à faire.

On peut ajouter que l'essai ultérieur prévu sur arbre de $5 \mathrm{~m}$ permettra une meilleure visualisation du phénomène.

M. Le Président. - Je remercie M. GUIDEZ de son exposé. 